\title{
ACUERDO TRANSPACÍFICO YASOCIACIÓN TRANSATLÁNTICA DE COMERCIO E INVERSIÓN. UN ANÁLISIS DEL NUEVO ESCENARIO COMER- CIAL INTERNACIONAL DESDE EL MERCOSUR
}

\author{
TRANSPACIFIC PARTNERSHIP AND TRANSATLANTICTRADE \\ AND INVESTMENT PARTNERSHIP. AN ANALYSIS OF THE NEW \\ INTERNATIONAL COMERCIAL SCENE FROM MERCOSUR
}

\author{
Julieta de San Félix ${ }^{1}$
}

\begin{abstract}
RESUMEN:
Durante los últimos años de la Administración Obama, Estados Unidos lideró la negociación de dos acuerdos que cambiarían el escenario comercial internacional: el Acuerdo Transpacífico de Cooperación Económica (ATCI) y la Asociación Transatlántica de Comercio e Inversión (ATP). Estos acuerdos, conceptualizados como megarregionales por su intención de crear zonas económicas integradas con socios de distintas áreas geográficas con el peso suficiente como para crear nuevas dinámicas en el comercio mundial, producirían, en el corto plazo, un doble impacto. Por un lado, profundizar las regulaciones vigentes en el ámbito de la Organización Mundial del Comercio (OMC), incluyendo cuestiones no acordadas como regulaciones tendientes a compras públicas o comercio electrónico. Por el otro, contener el crecimiento de China en el comercio internacional. La hipótesis que se explorará es que el rechazo al ATP y el actual paréntesis en las negociaciones del ATCI podría constituir una ventana de oportunidad para los países del Mercosur que no forman parte de estas mismas, los cuales, de mínima podrían mantener el status quo actual y, de máxima, podrían negociar en mejores términos el históricamente pen-
\end{abstract}

diente acuerdo con la Unión Europea, así como sus vinculaciones comerciales con Asia.

Palabras clave: Integración regional; Negociaciones comerciales; ATCI; ATP; Mercosur; OMC; Acuerdos comerciales; Acuerdos mega regionales; Aranceles.

\begin{abstract}
:
Among the last years of the Obama Administration, the United States leaded the negotiation of two agreements that would change the commercial global scene: the Transpacific Partnership and the Transatlantic Trade and Investment Partnership.

These agreements, conceptualized as megaregional by their intention to crate integrated economic zones with partners of different geographic areas with the sufficient weight to create new dynamics in the global commerce, would produce in the short term a double impact. On the one hand, to deepen the current regulations in the scope of the WTO, including areas not agreed as government procurement or electronic commerce. On the other hand, to contain China's growth.
\end{abstract}

1 Máster. Docente Adjunta e Investigadora en el departamento de Derecho y Ciencias Políticas. Universidad Nacional de la Matanza. Argentina. Correo electrónico: jdesanfe@gmail.com 
The hypothesis that will be explored in the article is that the rejection of the TPP and the current parenthesis in the TTIP negotiations could be a window of opportunity for Mercosur, which not being part of the negotiations, could at their minimum maintain the current status quo and, at their maximum, negotiate in better terms the historically pending agreement with the European Union, as well as improve its trade links with Asia.

Key words: Regional integration ; Commercial negotiations ;mega regional agreements; TTIP; TPP; Mercosur; WTO; Commercial agreement; tariffs.

\section{Introducción}

Existen más de 270 acuerdos comerciales vigentes en el marco de la Organización Mundial del Comercio (OMC). Todos sus miembros han firmado al menos un acuerdo comercial con otro socio. Un 10,68\% de estos acuerdos ha sido suscrito con antelación al Acuerdo de Marrakech por el que se establece el organismo en el año 1994 y un 13,10\% en los siete años de la Ronda Uruguay. El 76,20\% restante fue firmado a partir de la Ronda de Doha, en paralelo a lo que gran cantidad de analistas interpretan como un estancamiento del sistema multilateral (Ver figura 1).

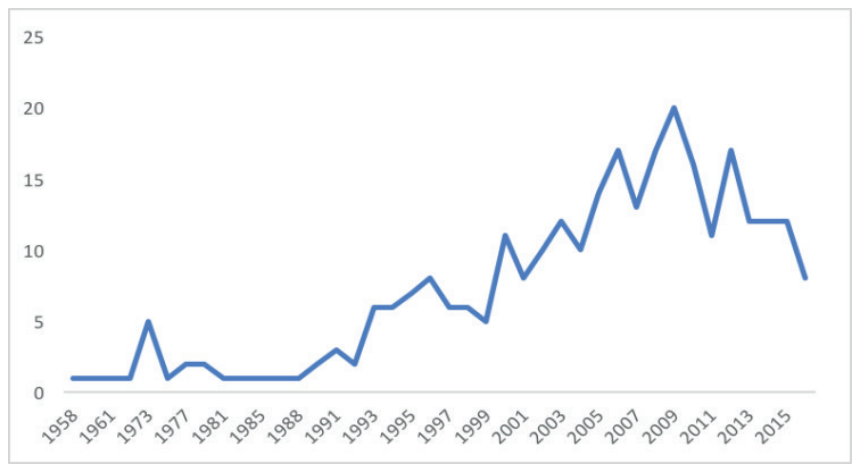

Figura 1. Acuerdos comerciales en el ámbito del GATT/GATS. Elaboración propia sobre la base de Datos de la OMC (2017). ${ }^{2}$

Estos acuerdos han tenido como objetivos manifiestos garantizar acceso preferencial a ciertos mercados, atraer inversiones, establecer relaciones pacíficas con otros países, incrementar el poder de negociación en la esfera internacional, consolidar reformas políticas domésticas, construir una alternativa a un sistema multilateral que ha logrado pocos acuerdos sustanciales en los últimos años ${ }^{3}$,

2 Se han tenido en cuenta las fechas de entrada en vigor de los acuerdos y se ha discriminado entre bienes y servicios cuando el documento fuese distinto. Asimismo, se han excluido los instrumentos posteriores a la entrada en vigor del acuerdo original.

3 Se exceptúa el Acuerdo sobre Facilitación del Comercio, que entró en vigor en 2017. 
profundizar la liberalización comercial existente, promover una dinámica comercial favorable a la integración y al encadenamiento de los sistemas productivos, entre otros ${ }^{4}$.

Estos acuerdos, que comprenden diversos modelos de integración ${ }^{5}$, son posibles en el ámbito multilateral y compatibles con la OMC en virtud del artículo XXIV del Acuerdo General sobre Aranceles Aduaneros y Comercio (GATT por sus siglas en inglés) y de los artículos V y V bis del Acuerdo General sobre el Comercio de Servicios (GATS por sus siglas en inglés), que habilitan la posibilidad del establecimiento de zonas de libre comercio, uniones aduaneras o procesos de liberalización del comercio de servicios, incluyendo la integración de los mercados de trabajo, siempre que se logre una cobertura sustancial de los mercados y que se respete el principio de no discriminación, incluido en las cláusulas de Nación Más Favorecida y Trato Nacional. Sin embargo, tanto el incremento en su número como su diverso tenor han despertado un debate académico y político sobre si este fenómeno favorece u obstaculiza las negociaciones en el ámbito de la $\mathrm{OMC}^{6}$.

Los entes defensores del multilateralismo destacan que el comercio internacional libre de barreras puede constituir un bien público mundial resultante de un intercambio entre el espacio de políticas nacionales que podrían habilitar la implementación de prácticas proteccionistas, a cambio del cual se podría llegar a un beneficio universal reduciendo los costos del comercio y aumentando la competitividad a largo plazo ${ }^{7}$.

Quienes defienden el regionalismo argumentan que la dificultad de la Ronda de Doha para la consecución de resultados ha habilitado a los países a perseguir sus políticas comerciales en forma unilateral, negociando con aquellos con que cuentan con una mayor complementariedad.

Sobre esta discusión surge el llamado "nuevo regionalismo", que supone un nuevo tipo de acuerdos comerciales recíprocos entre países desarrollados y países en desarrollo, que son impulsados por los intereses ofensivos de los más poderosos. Estos nuevos instrumentos, de los cuales el Tratado de Libre Comercio de América del Norte (TLCAN) constituye el paradigma, implican una mayor

4 Para un mayor desarrollo de los argumentos ver BID (2017); Cepal (2016).

5 Se sugiere revisarBalassa, Bela. 1982; y BID. 2017. Un debate sobre este tema da cuenta de si se trata de una diferencia de grado o de especie.

6 Dice el poema: “(...) Each is given a list of rules; a shapeless mass; a bag of tools. And each must fashion ere life is a flown; A stumbling block or a Stepping-Stone." (R.L. Sharpe).

Ver Baldwin (2014)

7 Ver a modo ilustrativo: https://www.wto.org/spanish/res_s/booksp_s/historywto_15_s.pdf 
cobertura que los acuerdos tradicionales (que se sustentaban estrictamente en cuestiones arancelarias) e incorporan temas cuyo tratamiento era históricamente resistido en el ámbito multilateral, por ejemplo, propiedad intelectual.

Con el comienzo de la Ronda de Doha, este "nuevo regionalismo" cede lugar al llamado "regionalismo del siglo XXI", que se sustenta en la mayor fragmentación internacional de los procesos productivos, visualizado en el rol cada vez más preponderante de las llamadas cadenas globales de valor. Estos encadenamientos productivos son posibles, a su vez, por la propia reducción de las barreras al comercio y a la inversión extranjera directa, los menores costos de transporte y los adelantos en las tecnologías de la información y la comunicación, que han posibilitado la deslocalización de buena parte de la producción, propiciando una mayor competitividad y que ha tenido como correlato "el aumento de contenido importado de los productos exportados” (Cepal, 2016, p. 12).

El regionalismo del nuevo siglo se centra menos en temas regulatorios -aunque no los desestima- y más en la armonización de las políticas internas que crean barreras al comercio, incrementan el costo de hacer negocios en el exterior y limitan la movilidad y el acceso a las inversiones. Avanza al margen de la OMC y podría consolidar las asimetrías de poder subyacentes al multilateralismo a través de la erosión de su propósito de bien público mundial y la exclusión de determinados países de su alcance.

Se superan, así, las tradicionales negociaciones arancelarias y se comprenden cada vez más cuestiones sui generis, dando origen a los denominados temas OMC plus (OMC+) y OMC extra (OMC-x). De acuerdo con Horn, Mavoradis y Sapir (2009), se clasifican como OMC+aquellos compromisos construidos sobre la base de temas ya acordados en el nivel multilateral. Por ejemplo, reducciones arancelarias. Los OMC extra son aquellos compromisos que abarcan temas que van más allá del actual mandato de la OMC. Por ejemplo, estándares laborales. Los primeros son propios del nuevo regionalismo mientras que los segundos lo son del regionalismo del siglo XXI.

De acuerdo con Bouzas y Zelicovich:

El dato más relevante de la explosión de regionalismo en las tres últimas décadas no es el número de acuerdos negociados sino su utilización estratégica por parte de actores clave que han visto fuertemente limitada su capacidad de modelar las reglas internacionales a través de los canales tradicionales que ofrecía la institucionalidad multilateral. En las nuevas condiciones del régimen multilateral el "poder de avanzar solo" ya no se refleja en la capacidad de influir de manera directa sobre su contenido sino en la construcción 
de regímenes regulatorios alternativos que eventualmente se conviertan en referencia para el resto de la comunidad internacional, anulando de facto la relevancia del status quo multilateral. (Bouzas y Zelicovich, 2014, p. 979)

Los llamados acuerdos megarregionales condensan en sus textos los contenidos del regionalismo del siglo XXI, promoviendo la creación de nuevas dinámicas que podrían afectar sustancialmente la configuración del sistema internacional de comercio. En virtud de ello, y en un mundo en el cual la dinámica comercial se encuentra en un proceso de cambio estructural, se propone analizar las oportunidades derivadas del curso de las negociaciones del ATCI y del ATP en el Mercosur.

\section{Diseño metodológico}

La pregunta de investigación que guió el presente trabajo fue: ¿De qué modo las negociaciones comerciales internacionales, especialmente el Acuerdo Transpacífico y la Alianza Transatlántica de Comercio e Inversión, podrían afectar el desempeño del Mercosur en el sistema comercial internacional)? Asimismo, se procuraron evaluar las estrategias de regionalismo a disposición de los órganos tomadores de decisión en el bloque sudamericano en el nuevo escenario internacional.

Del amplio espectro de acuerdos que con el rótulo de "mega-regionales" se encuentran en proceso de negociación o se han concluido recientemente, se seleccionaron como unidades de análisis el ATP y el ATCI, porque cuando las negociaciones de aquellos fueron lanzadas involucraban a los dos mayores socios comerciales del bloque: Estados Unidos y la Unión Europea. Hoy, China ha desplazado a Estados Unidos como primer socio comercial, por lo que un estudio futuro debería incluir el análisis en profundidad del potencial impacto de la Asociación Económica Regional Integral sobre el Mercosur.

Para analizar la pregunta planteada, se partió de un escenario global signado por tres factores de contexto: la crisis de 2008 tras la cual muchos países no han logrado retornar a los niveles de crecimiento anteriores a su desenlace, el aludido estancamiento en las negociaciones comerciales multilaterales en el ámbito de la OMC y los cambios políticos supuestos por la elección de Donald Trump como Presidente de Estados Unidos y la decisión del Reino Unido de retirarse de la Unión Europea.

En el trabajo, de índole eminentemente descriptiva, se han tomado como variables independientes los acuerdos megarregionales (en especial, el ATP y el 
ATCI), y como variable dependiente las oportunidades del Mercosur en virtud del devenir de estos mismos. Se utilizan como indicadores de análisis el contenido de los textos negociados, el curso de las negociaciones, los acuerdos regionales concluidos y en negociación, la inserción del bloque en el comercio internacional y su canasta exportadora hacia tres destinos: Estados Unidos, la Unión Europea y China.

El trabajo partió de un relevamiento de fuentes primarias documentales (documentos producidos por organismos internacionales y centros de estudio especializados, discursos y declaraciones a la prensa) y de entrevistas semiestructuradas a actores clave, desarrolladas entre enero de 2016 y abril de 2017, a efectos de evaluar la percepción de los órganos tomadores de decisión sobre la problemática. Estas fuentes fueron complementadas con un análisis de fuentes secundarias, periodísticas y bibliográficas, para arribar a las conclusiones exploratorias presentadas al final del artículo.

\section{Acuerdos megarregionales: Acuerdo de Asociación Transpacífico y Acuerdo Transatlántico sobre Comercio e Inversión}

Ante el virtual estancamiento de la OMC durante la Ronda de Dohay acompañando la caída del comercio internacional tras la crisis de 2008/2009, muchos países comenzaron a negociar una nueva generación de acuerdos comerciales preferenciales, llamados megarregionales, entre los que se encuentran el Acuerdo de Asociación Transpacífico (ATP), la Asociación Económica Regional Integral (RCEP, por sus siglas en inglés) ${ }^{8}$ y el Acuerdo Transatlántico sobre Comercio e Inversión (ATCI). Estos tres acuerdos involucran al 61\% de la población del planeta, al 70,8\% de la inversión extranjera directa (IED), al $70 \%$ del comercio internacional ${ }^{9}$ y al $81,22 \%$ del PBI mundial. Solo el ATP y el ATCI comprenden al 17,8\% de la población mundial, el 55\% de la IED, el $60 \%$ del comercio internacional y casi al $60 \%$ del producto bruto ${ }^{10}$. A estos, muchos analistas $^{11}$ suman, por su magnitud, el Acuerdo entre la Unión Europea y Canadá, entre la Unión Europea y Japón, y la Alianza del Pacífico.

8 El RCEP es una negociación para un acuerdo de libre comercio lanzada en 2012 que incluye a China, junto a los miembros de la Asociación de Naciones del Sudeste Asiático (Brunei, Camboya, Indonesia, Laos, Malasia, Myanmar, Filipinas, Singapur, Tailandia y Vietnam) y los seis países con los cuales ASEAN tiene acuerdos de libre comercio (además de China, Australia, India, Japón, República de Corea y Nueva Zelandia)

9 Inversión Extranjera Directa. Flujos netos.

10 Elaboración propia sobre datos del Banco Mundial (2017). Datos de 2015.

11 Ver Rensmann, t. (2017) 
Todos estos acuerdos han sido conceptualizados como megarregionales por su intención de crear zonas económicas integradas con socios de distintas áreas geográficas con el peso suficiente como para promover un cambio sistémico en el comercio internacional. Surgen en un contexto en el que, con dificultades para recuperar un crecimiento sostenido tras la crisis de 2008 (ver figura 2), los países buscan incorporar una mayor productividad a través de la integración en cadenas globales de valor ${ }^{12}$ que pueden recrear nuevas dinámicas, poniendo incluso en cuestión las ventajas comparativas tradicionales.

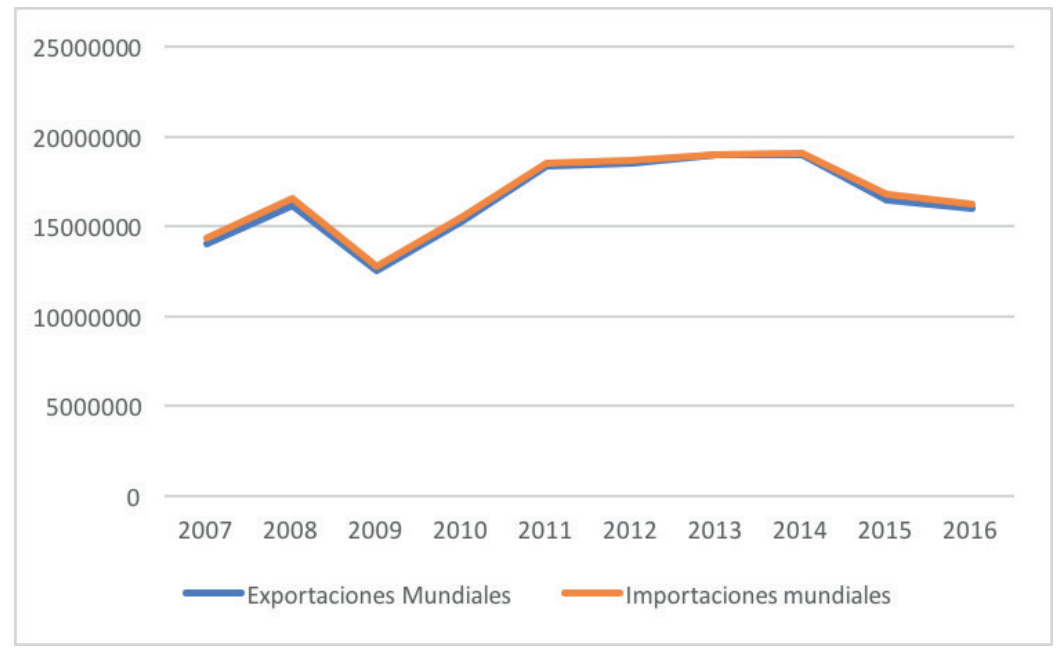

Figura 2. Evolución del volumen del comercio internacional de bienes (en precios corrientes, u\$S a 2010). Elaboración propia sobre la base de datos de la OMC (2017).

En este escenario, a partir del año 2008 se comienzan a negociar acuerdos macro que:

buscan armonizar, o al menos hacer compatibles, las reglas mediante las cuales operan las distintas fábricas mundiales', facilitando las operaciones de las empresas multinacionales que tienen operaciones en América del Norte, Europa y Asia oriental y sudoriental. (Rosales et al., 2013, p. 15)

En palabras de Carrión Fonseca:

12 Se denominan cadenas globales de valor al "conjunto de actividades o etapas de producción para la elaboración de bienes y servicios, desde la inicial de producción de insumos hasta la final de consumo, que se realizan en dos o más países.” (Tello, 2015) 
En primer lugar, los acuerdos megarregionales son $<$ asociaciones de integración entre países y/o regiones que representan una porción importante del comercio internacional y de la inversión extranjera directa (IED) y en las cuales uno o más de los socios son líderes y/o funcionan como centros o plataformas para las cadenas de valor global> (Meléndez en World Economic Forum 2014:1). En segundo lugar, estos tienen como objetivos crear espacios económicos de largo alcance que trascienden la lógica bilateral de la mayoría de los acuerdos regionales actuales (Cepal 2013a). En tercer lugar, la agenda de negociación abarca una serie de áreas que van más allá de las abordadas en los acuerdos de la OMC. Por último, los acuerdos megarregionales reflejan también el interés de Estados Unidos y de la Unión Europea de continuar manteniendo un rol decisivo en la creación de reglas de comercio e inversión. (Carrión Fonseca, 2015, p. 15)

La figura 3 ilustra la proporción entre el país con mayor PBI de cada uno de estos acuerdos y el resto de los países agregados. A excepción del ATCI que demuestra un mayor equilibrio entre los socios de ambos lados del Atlántico, tanto el ATP como el RCEP presentan una fuerte asimetría entre su socio más poderoso (Estados Unidos en el primero y China en el segundo) y el resto de los países involucrados. En ambos casos, el peso del país más poderoso es tan importante que la dinámica comercial resultante podría ser absolutamente distinta sin ellos. Incluso, en un tratado entre los 28 países de la Unión Europea (UE) y Estados Unidos (EEUU) resulta también desproporcionado el peso del país americano.

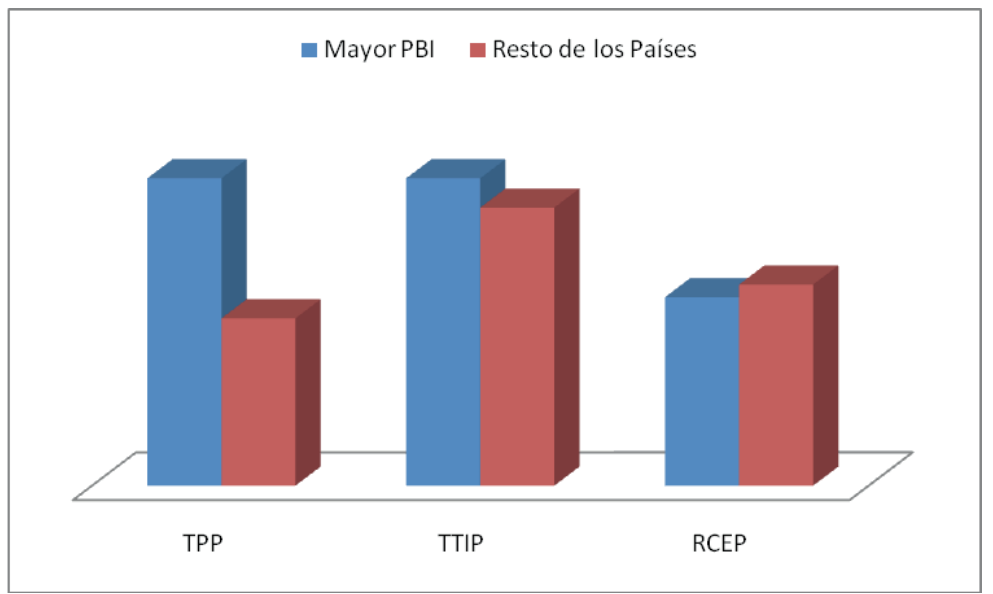

Figura 3. Peso relativo del país con mayor PBI respecto al resto de los países. Elaboración propia sobre la base de datos del Banco Mundial. PBI 2015 estimado a precios corrientes. 
No se trata de los acuerdos regionales clásicos basados eminentemente en liberalización arancelaria, sino que incorporan disposiciones $\mathrm{OMC}+\mathrm{y} \mathrm{OMCx}$, lo cual encuentra correspondencia con el "regionalismo del siglo XXI", especialmente en lo que respecta a la combinación entre las reglas del juego y la voluntad de una nueva gobernanza internacional del comercio.

En palabras de la Cepal:

Las complejas relaciones de comercio e inversión que se dan al interior de las redes internacionales de producción requieren un entorno normativo propicio para su desarrollo. Específicamente, se requieren disciplinas que garanticen: i) el libre flujo de bienes, la información, las personas y los capitales involucrados en el funcionamiento de las cadenas de valor; y ii) la protección de los derechos de propiedad, tanto tangible como intangible, que poseen las empresas multinacionales participantes en dichas cadenas. (Rosales et al., 2013, pp. 13-14) ${ }^{13}$

Por último, estos acuerdos surgen en un escenario internacional signado por la excepcionalidad de China, que representa el 15\% del PBI mundial y que hasta 2014 ha registrado tasas de crecimiento superiores al 7\% anual, aunque en los últimos años muestra una desaceleración también evidente en sus indicadores comerciales. No obstante, la buena performance de sus indicadores sociales y su incorporación a la OMC, evidencian un mercado de especial interés y alta potencialidad que representa en la actualidad al primer importador y segundo exportador mundial de bienes ${ }^{14}$, en una región de gran dinamismo comercial.

En suma, tanto el ATP como el ATCI revelan una estrategia comercial ofensiva de EEUU y la Unión Europea signada por la voluntad de asegurar las cadenas de valor, acceder en forma segura y competitiva a las materias primas, contener el crecimiento de China, y la profundización de las regulaciones de la OMC, a falta de avances concretos en el plano multilateral. Iniciativas como el RCEP y la Alianza del Pacífico surgen como reacción a estas dinámicas.

\subsection{Tratado de Asociación Transpacífica}

El ATP fue firmado en Auckland el 4 de febrero de 2016 por los gobiernos de Australia, Brunei, Canadá, Chile, Estados Unidos, Japón, Malasia, México, Nueva Zelanda, Perú, Singapur y Vietnam, países que representan el 37\% del

13 Una de las cuestiones más debatidas en estos acuerdos ha sido a constitución de las instancias arbitrales. Ver, Warren, Elizabeth (2015) y Beattle, Bryain (2017).

14 Datos Cepal, 2015. 
PBI mundial, el 34\% de las inversiones extranjeras directas, el 26\% del comercio internacional y el $11 \%$ de la población de todo el planeta ${ }^{15}$.

En 30 capítulos, el Acuerdo incorpora disposiciones sobre aranceles y acceso a mercado, reglas y procedimientos de origen, textiles, administración de aduanas y facilitación del comercio, medidas comerciales correctivas, medidas sanitarias y fitosanitarias, barreras técnicas al comercio, inversiones, comercio transfronterizo de servicios, servicios financieros, ingresos temporarios para personas de negocios, telecomunicaciones, comercio electrónico, compras públicas, competencia, empresas de propiedad del estado y monopolios designados, propiedad intelectual, empleo, ambiente, cooperación y fomento de capacidades, competitividad y facilitación de negocios, desarrollo, pequeñas y medianas empresas, coherencia regulatoria, transparencia y medidas anticorrupción, normas administrativas e institucionales, solución de controversias, y excepciones.

Las negociaciones se sentaron sobre las bases del Acuerdo Estratégico Transpacífico de Asociación Económica (Pacific 4-P4) que, suscrito en 2005, constituyó el primer tratado plurilateral que vinculaba a países de América Latina, Asia y Oceanía. Más allá del P4, las 12 economías signatarias se encuentran entrelazadas por una densa red de acuerdos comerciales. Diez de estos países cuentan contratados de libre comercio con al menos la mitad de los miembros del ATP, y más del 80 por ciento del comercio ya está abarcado por acuerdos bilaterales. Los países con mayor volumen de comercio intra ATP son Brunei, Canadá y México.

Poco más de un tercio (36\%) de todas las relaciones bilaterales posibles no están abarcadas por un acuerdo. Según Rosales y otros (2013), las más importantes de estas relaciones son aquellas que vinculan a los Estados Unidos con Japón, Malasia y Vietnam.

En materia de acceso a mercados, Cepal (2016) ha estimado que en 2014 el promedio arancelario aplicable era del 2,7\%, por lo cual la ganancia para los países miembros sería genéricamente marginal. La excepción la constituye el sector agrícola y alimenticio, con aranceles en la actualidad superiores al 15\% y para los cuales se han obtenido compromisos de apertura profundos. Por su parte, las reglas en torno a acumulación de origen, que permite a los productos de un país miembro ser objeto de una transformación posterior o ser añadidos a productos en otros países miembros como si fueran originarios de este último país, fomentarían una mejor inserción en las cadenas globales de valor para quienes

15 Elaboración propia sobre datos del Banco Mundial (2017). Datos de 2015. 
pudieran transformar sus sectores productivos aunque podría crear externalidades negativas para los que no fueran parte del acuerdo al tiempo que una perpetuación de términos de intercambio desfavorable para aquellos miembros que no pudieran adaptarse.

En materia de inversiones, el ATP introduce innovaciones al mecanismo de solución de controversias, incorpora una revisión expedita de las denominadas controversias frívolas, puede adjudicarse el pago de los costos respectivos a la empresa que inició el caso, define reglas de conducta para los árbitros y explicita, entre otras, que en toda reclamación la carga de la prueba caiga sobre el reclamante. Este punto ha sido criticado con el argumento de que no se ha incluido una opción de examen preliminar que permitiese a los gobiernos de los países involucrados en una denuncia decidir si esta pudiese proceder y tampoco incorpora una instancia de apelación, lo que crearía un sesgo a favor de los inversores.

Uno de los capítulos más controvertidos de las negociaciones fue el ateniente a los derechos de propiedad intelectual. Las regulaciones señalan una tendencia al alza de la protección respecto a los niveles mínimos establecidos en el Acuerdo sobre los Aspectos de los Derechos de Propiedad Intelectual (ADCIP). La nueva regulación podría permitir a titulares de patentes extender el monopolio que estas les confieren mediante innovaciones menores, lo que a su vez podría dilatar la difusión masiva de los nuevos adelantos tecnológicos. El Tratado incluye también previsiones novedosas tendientes a la protección de los derechos de autoría en el entorno digital. Se incorporan disposiciones sobre exclusividad de los datos de prueba requeridos para la comercialización de productos farmacéuticos y biológicos. Asimismo, se prohíbe a las autoridades sanitarias de los países otorgar permisos de comercialización de un producto farmacéutico genérico hasta la expiración de la patente respectiva que favoreciese la versión original. Por último, requiere a los países miembros que accedan al Acta de 1991 del Convenio Internacional para la Protección de Nuevas Variedades de Plantas, lo cual despierta preocupación ante la posible concentración de los derechos de obtentor en unas pocas multinacionales.

También, introduce regulaciones al comercio electrónico y la economía digital, establece un marco legal orientado a igualar las condiciones de competencia entre las empresas estatales y privadas en temas de comercio e inversión, actuando conforme las consideraciones comerciales vigentes, como, por ejemplo, otorgar el estatus de trato nacional a las empresas de otros miembros, con la posibilidad de que se excluyeran a algunas empresas de estas obligaciones. Por último, se introducen también regulaciones laborales y ambientales, muchas de 
las cuales han requerido el compromiso de los países que estaban por debajo de esos estándares de adaptar su legislación en conformidad ${ }^{16}$.

\subsection{Acuerdo Transatlántico de Comercio e Inversión}

El ATCI aún se encuentra en la mesa de negociaciones entre la Comisión Europea y el gobierno de EE. UU. De concluirse comprendería aproximadamente el $60 \%$ del PBI mundial, un tercio del comercio internacional de bienes y servicios y un mercado de más de 800 millones de sujetos consumidores, que representan poco más del 11\% de la población mundial.

Este acuerdo se viene negociando desde el año 2013, como fruto de una intensa red de acercamientos transatlánticos previos. Se estructurará sobre tres pilares: (1) acceso al mercado de bienes y servicios, inversiones y contratación pública; (2) convergencia regulatoria y barreras no arancelarias, presentes en la agenda de la $\mathrm{OMC}+$; (3) gobernanza del comercio mundial en términos sistémicos, incluyendo temas OMCX.

Según el impacto de sostenibilidad encargado a la Comisión Europea (Ecory 2017), las tasas arancelarias entre ambos países son bajas en promedio (2,2\% para EEUU y 3,3\% para la Unión Europea), aunque alcanzan picos notables en determinados productos. Mientras que la UE carga más la importación de vehículos a motor o alimentos elaborados, EE. UU. tiene mayores tasas para material ferroviario, llegando a un fuerte proteccionismo en algunos productos agrícolas. A modo ilustrativo, el arancel al tabaco europeo es del 350\%.

El principal objetivo, según lo ha declarado el Comisionado de comercio de la UE, es remover todas las barreras que van más allá de las aduanas, como diferencias en regulaciones técnicas, estándares y certificaciones ${ }^{17}$.

Según la Comisión Europea (2015), el comercio bilateral de bienes llegó a 517,1 mil millones de euros en 2014 y los servicios acumularon los 375,7 mil millones de euros en el mismo período. EE. UU. es en la actualidad el principal socio comercial de la Unión Europea. Asimismo, es el primer destino de la inversión extranjera directa europea, reporta en 2014 alrededor de 225,2 mil millones de

16 Por ejemplo, en el marco del artículo 19 sobre derechos laborales del ATP, Vietnam y Estados Unidos han aprobado un acuerdo bilateral por el cual el primero se compromete a garantizar el derecho a la sindicalización.

1721 de febrero de 2013. Discurso del Comisionado de Comercio de la UE, Karel De Gucht. Disponible en: http://trade.ec.europa.eu/doclib/docs/2013/february/tradoc 150581.pdf 
euros, y viceversa (las inversiones estadounidenses en Europa significaron unos 421,2 mil millones de euros).

La citada evaluación de impacto estima que el acuerdo podría aumentar el PBI europeo en un $0,5 \%$ anual y el de EEUU en un $0,4 \%$ anual y generar dos millones de empleos en Europa. Un 80\% de las ganancias potenciales resultarían de la reducción de los costos derivados de las barreras burocráticas y regulatorias y de la liberalización de servicios, así como del mercado de compras y contrataciones públicas.

Subsisten temas de potencial conflicto como las sensibilidades en torno a ciertas regulaciones como bienes culturales, uso de hormonas, químicos o antibióticos en la producción alimenticia o el consumo de transgénicos.

En el año 2015, la Comisión adoptó una nueva estrategia de comercio que plantea los acuerdos comerciales como instrumentos que le permiten una mejor inserción en las cadenas globales de valor. Para ello, propone priorizar el comercio de servicios, facilitar el comercio digital, fomentar la innovación, promover la movilidad de profesionales y personal directivo de empresas, reforzar la cooperación internacional en materia regulatoria, asegurar una administración aduanera eficiente y asegurarse también el acceso a las materias primas y recursos energéticos. Se persigue un nuevo formato de acuerdos que incluye cláusulas laborales, medioambientales y políticas.

Destacan la importancia de concluir el mandato de Doha, proponer nuevas reglas de gobernanza del comercio y fortalecer el sistema multilateral de comercio. La Comisión también afirma que utilizará el antecedente de acuerdos logrados en el plano bilateral o regional para enfrentar los desafíos de la OMC. Se observa, en esta estrategia, la intención de la UE de avanzar en el plano bilateral para configurar nuevas reglas multilaterales, coincidentemente con el regionalismo del siglo $\mathrm{XXI}$.

\subsection{Impacto sistémico de los acuerdos megarregionales}

Estos acuerdos tendrán un impacto sistémico significativo en el comercio internacional, favoreciendo determinados flujos comerciales y propiciando determinados encadenamientos productivos. Ello provocará externalidades para aquellos países que no hayan ingresado en estas preferencias, que dependerán del volumen comerciable con sus socios, la competitividad de cada economía y la capacidad de adaptarse a las nuevas reglas. 
A su vez, y como parte de la estrategia ofensiva de los países más influyentes, estos acuerdos provocarán una modificación de las reglas internacionales del comercio, sin necesidad de alterar el marco de la OMC; pero, muy probablemente, promoviendo a largo plazo una variación en las reglas de la institución. Como consecuencia, los acuerdos serán cada vez menos estrictamente comerciales en el marco GATT y GATS, e incluirán regulaciones de enforcement, tópicos que se están discutiendo en la OMC aún sin consenso y otras disposiciones no tarifarias como cláusulas de derechos humanos, ambientales y sociales.

Subyace al impacto de los acuerdos megarregionales la cuestión sobre si el éxito de estos constituye un riesgo para el sistema multilateral de comercio al hacer prescindible la OMC. Como se ha argumentado, los países más poderosos han buscado invertir la regulación del sistema comercial internacional: son estos acuerdos megarregionales los que con su impacto configuran las reglas del resto de los actores, incluso de aquellos que no hayan ingresado. Son sistémicos en tanto y en cuanto no pueden ser obviados y promoverán cambios sustanciales.

Ambos acuerdos, como se refirió anteriormente, buscan también contener el ascendente peso de la República Popular China en el comercio internacional, en especial a partir de su incorporación como miembro pleno de la OMC, sobre la cual tanto el gobierno de EE. UU. como la UE ha planteado serios interrogantes por las denunciadas políticas de dumping que darían competitividad al gigante asiático. La rápida reacción China constituyó el inicio de las negociaciones del RCEP en 2014.

\section{Los acuerdos megarregionales en el nuevo escenario geopolítico y comercial mundial}

Los recientes cambios geopolíticos en el Atlántico Norte están provocando un abrupto giro comercial tanto en EE. UU. como, con matices, en Europa.

La salida del Reino Unido de la Unión Europea, el ascenso de movimientos euroescépticos, y la agenda de política exterior denominada "America First"18", proclamada por el presidente de EE. UU., han modificado el mapa político y comercial vigente.

El Brexit ha funcionado como un catalizador de demandas contenidas en la opinión pública europea, especialmente aquellas relativas al empleo. Este resultado favoreció el crecimiento de movimientos antieuropeístas, euroescépticos

18 "Primero América". Disponible en: https://www.whitehouse.gov/america-first-foreign-policy 
y antiglobalización. Ante el riesgo de un repliegue proteccionista, surgen dos interrogantes en el seno de la UE. Por un lado, ¿cómo evitar nuevos desmembramientos? Por el otro lado, ¿cómo democratizar y visibilizar los beneficios del comercio? La opción por una mayor transparencia en las negociaciones y la aprobación de una nueva estrategia comercial fueron las respuestas a estos interrogantes.

Del otro lado del Atlántico, los anuncios de campaña del presidente estadounidense en lo que respecta a la salida del Acuerdo de Asociación Transpacífica, a una renegociación del TLCAN y al cambio en su estrategia comercial que privilegia políticas proteccionistas comenzaron a concretarse en los primeros días de gobierno. El 23 de febrero de 2017, el gobierno de Estados Unidos emite un Memorándum Presidencial ${ }^{19}$ en el que retira la firma del Acuerdo Transpacífico.

Hasta entonces, el acuerdo había sido ratificado únicamente por Japón, país que ingresa tardíamente a las negociaciones, pero que emite una señal al ratificarlo en diciembre de 2016. En mayo de 2017, Nueva Zelanda se convierte en el segundo país en hacerlo.

El Tratado hubiese entrado en vigor una vez que lo ratificas en los doce países signatarios o si, en un plazo de dos años, lo firmasen al menos 6 países que representasen el $85 \%$ del PBI de los doce originales. Dado que EE. UU. comprende el 65,3\% del PBI de este bloque, retiene su poder de veto.

Ante este escenario, los países signatarios han buscado impulsar un acuerdo sin EE. UU.; Japón ${ }^{20}$ y Australia han sido los más comprometidos con esta estrategia. Sin embargo, en materia comercial, los países del ATP están ya vinculados entre sí por una red de acuerdos bilaterales y plurilaterales que, de hecho, ya implica concesiones importantes en materia de acceso a mercado. Casos extremos de liberalización previa lo constituyen Chile y Singapur con acuerdos con casi todos los socios. Del otro lado se encuentran Canadá o México, que ampliarían sustancialmente su liberalización arancelaria. Coincidentemente, países como Chile han optado por fortalecer otros mecanismos como la Alianza del Pacífico ${ }^{21}$.

19 Memorándum Presidencial concerniente al retiro de la firma de los Estados Unidos de las negociaciones y el Acuerdo Transpacífico (23/01/17)

$20 \mathrm{http}$ //foreignpolicy.com/2017/04/24/japan-wants-to-revive-trans-pacific-partnership-even-withoutunited-states-asia-trade-agreements/

21 http://www.lavanguardia.com/politica/20170315/42888152799/sin-tpp-alianza-pacifico-fortalecerol-de-interlocutor-con-paises-asiaticos.html 
Las opciones planteadas en el nuevo escenario son: (1) enmendar la cláusula de ratificación y continuar con un ATP11; (2) promulgarlo de manera provisional; (3) renegociarlo y relanzarlo, buscando que se incorpore Estados Unidos; (4) avanzar en el esquema bilateral; (5) aceptar la situación y volcar los esfuerzos al fortalecimiento de la $\mathrm{OMC}^{22}$.

El giro político estadounidense frente al ATP ha despertado la incertidumbre también sobre el futuro del ATCI. De hecho, desde el cambio de gobierno en EE. UU. no se han llevado a cabo nuevas rondas y ciertos analistas ${ }^{23}$ descartan una inminente modificación en las condiciones de la negociación.

Todo esto en un escenario en el cual la economía mundial aún no recupera los niveles de crecimiento anteriores a la crisis del 2008 y que tras un leve repunte, a partir de 2010, vuelve a ver caer el volumen del comercio internacional en el bienio 2015 - 2016.

\section{El Mercosur ante el regionalismo del siglo XXI}

El Tratado de Asunción, suscrito en 1991 por Argentina, Brasil, Paraguay y Uruguay dio origen al Mercado Común del Sur (Mercosur), que implicaría la libre circulación de bienes, servicios y factores productivos entre sus miembros a través de la eliminación de los derechos aduaneros y otras restricciones no arancelarias, el establecimiento de un arancel externo común, la adopción de una política comercial común con relación con terceros Estados o bloques y la coordinación de posiciones en foros económico- comerciales, políticas macroeconómicas y sectoriales y el compromiso de las partes de armonizar sus legislaciones para fortalecer la integración.

Con una concreción dispar de sus objetivos iniciales, se ha sumado Venezuela al bloque ${ }^{24}$ y se encuentra en proceso de ratificación la incorporación de Bolivia.

22 Recientemente, en paralelo a una reunión de la ASEAN, se dieron cita representantes de los once países remanentes en el ATP, y acordaron continuar con el instrumento

23 González (2017).

24 El Protocolo de Adhesión de Venezuela al Mercosur preveía un plazo de cuatro años a partir de la entrada en vigor de dicho instrumento, para la incorporación del acervo normativo del bloque. El 13 de septiembre de 2016 los Cancilleres de los Estados miembros otorgaron a dicho país un plazo complementario para la adopción de la normativa, el cual vencía el día 1 de diciembre de 2016 . El 2 de diciembre, dictaminando un estado de incumplimiento, cancilleres de Argentina, Brasil, Paraguay y Uruguay decidieron el cese de los derechos inherentes a la condición de Estado parte para Venezuela. E1 5 de agosto de 2017, los cancilleres de Argentina, Brasil, Paraguay y Uruguay emitieron una De- 
Siguiendo un patrón de regionalismo abierto hasta la década de 2000, que fue sustituida por una fase en la cual se han priorizado las negociaciones sur-sur hasta el año 2015, el bloque se ha mantenido al margen de las negociaciones megarregionales descriptas.

Mercosur cuenta con acuerdos comerciales vigentes con socios extra latinoamericanos como Israel, India y la Unión Aduanera de África del Sur (SACU) $\mathrm{y}$ tiene en proceso de ratificación de acuerdos de libre comercio con Egipto y Palestina. Ha firmado instrumentos para la formulación de tratados de libre comercio con Marruecos, Sudáfrica y Jordania (en vigor), Turquía y Siria (pendientes). Por último, ha entablado conversaciones institucionalizadas para la firma de un acuerdo comercial con Corea del Sur.

En lo que respecta a la región y con depósito en la Asociación Latinoamericana de Integración (ALADI), el Mercosur ha suscrito Acuerdos de Complementación Económica con Chile, Bolivia, México, Perú, Colombia, Ecuador y Venezuela. Asimismo, ha iniciado conversaciones para la firma de estos acuerdos con Panamá y República Dominicana.

La evolución del comercio del Mercosur en los últimos años ha seguido un patrón similar al mundial, con volúmenes de mercancías que cuesta mantener cercanos al pico registrado en el año 2007 (ver figura 4). La crisis política en Brasil y Venezuela, así como la desaceleración de China, no favorecen un pronto repunte de esta situación. La caída del volumen y monto del comercio fue más pronunciada en este bloque, porque sus ventas están más concentradas en productos básicos, y por la caída en el precio de las materias primas. Los principales cinco productos exportados en 2017 fueron minerales, oleaginosas y granos, residuos de la industria alimenticia, derivados militares, y carnes; productos que representaron el $40,5 \%$ de las exportaciones totales.

Otro problema que han enfrentado estos países son las tensiones inflacionarias. Por último, la inversión extranjera directa (IED) sufre una contracción del $14,9 \%$ en 2015 , en un contexto en el que los flujos de IED mundiales aumentaron en aproximadamente un $38 \%{ }^{25}$.

cisión por la cual aplican a Venezuela el Protocolo de Ushuaia sobre Compromiso Democrático del Mercosur y suspendieron a dicho país de sus derechos y obligaciones de participación en el bloque.

25 Datos Cepal, 2016. 


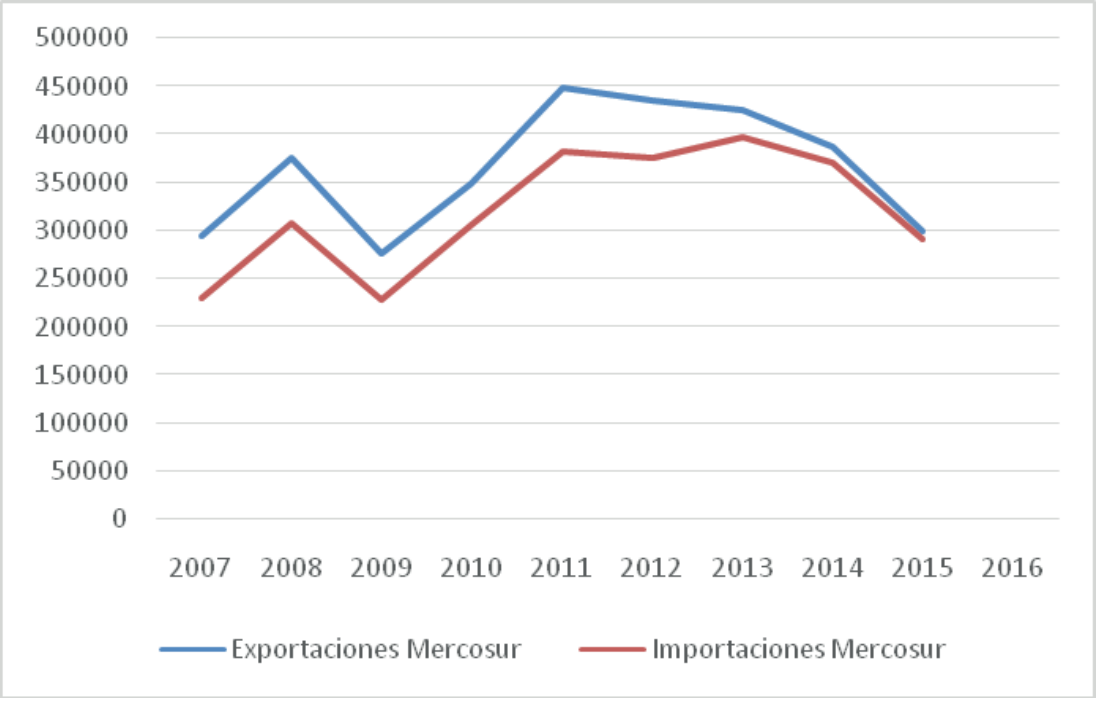

Figura 4. Evolución del comercio del Mercosur en bienes (miles de dólares). Elaboración propia sobre la base de datos de Comtrade (2017).

\subsection{Negociaciones con Europa}

Las negociaciones para la consecución de un acuerdo entre la Unión Europea y el Mercosur se lanzaron en el año 2001 y, desde entonces, se intercambiaron ofertas de liberalización en dos oportunidades. Mientras que se han registrado avances sustanciales en los capítulos de diálogo político y cooperación, el comercio continúa siendo problemático debido a las sensibilidades. Por su parte, por el principio de single undertaking26 desde el cual se llevan las negociaciones, no se firmará ningún texto hasta que todos los capítulos hayan sido acordados.

Una primera fase de negociaciones fue interrumpida en 2004 luego de un primer intercambio de ofertas insatisfactorio para las partes, a la que siguió un relanzamiento infructuoso de estas en el año $2010 \mathrm{y}$, finalmente, un avance desde 2015 en paralelo a los cambios de gobierno en el Mercosur, al Brexit y al avance de las descriptas negociaciones megarregionales. Se observa la dependencia del

26 Principio que establece que, no existirá acuerdo hasta que todas las partes que componen la negociación hayan sido acordadas individualmente. 
curso de las negociaciones en relación con la coyuntura tanto nacional como internacional.

En 2016 se produce un nuevo intercambio de ofertas, también insatisfactorio para ambas partes, producto de presiones domésticas de ambas partes. En el caso de la UE, excluyó de su oferta a bovinos y etanol, lo que provocó el rechazo del Mercosur.

A pesar de esto, las rondas continuaron regularmente y en marzo de 2017 se consensuó un cronograma intensivo de negociaciones, a efecto de llegar a la firma del acuerdo antes del cambio de la Comisión en 2019. Se ha dicho que el Mercosur aprovecharía la Ministerial de la OMC en Buenos Aires en diciembre de 2017 para hacer un anuncio ${ }^{27}$.

Más allá de las cuotas de acceso a mercado, un punto central de la discusión arancelaria lo constituyen los plazos de desgravación.

Cuando las negociaciones se lanzaron en 2001 la Unión Europea había concluido unos quince acuerdos comerciales de distinto tenor. Hoy, la UE registra 45 convenios. En quince años, la cantidad de acuerdos comerciales de la UE se triplicó. Pero no se trata exclusivamente de una cuestión numérica. Progresivamente se han ido incorporando disposiciones que superan el ámbito arancelario (OMC+ y OMCx), avanzando hacia el "regionalismo del siglo XXI". Esto ha repercutido en las negociaciones con el Mercosur, en las que se ha incorporado un capítulo referente a desarrollo sostenible que incluye cláusulas de cuidado ambiental y regulaciones sindicales.

El impacto de un acuerdo sería considerable para ambos bloques, según demuestran los actuales intercambios y como lo han referido los actores consultados. El valor del comercio interbloque representó en 2016 para el Mercosur el $15,75 \%$ de su comercio global; pero para la UE significó poco más del 5\%. Por su parte, en los últimos años, la Unión Europea vio desplazada su supremacía comercial en la región en favor de China. Esta situación podría revertirse de firmarse el acuerdo.

El valor total de las exportaciones del Mercosur a la Unión Europea se encuentra hoy en repliegue. Sin embargo, la canasta exportadora está mucho menos concentrada que en otros casos. Los cinco principales productos exportados a

27 Iprofesional. Malcorra desea anunciar un acuerdo de libre comercio Mercosur-UE en diciembre. 20/04/2017. Disponible en: http://www.iprofesional.com/notas/248691-Malcorra-desea-anunciarun-acuerdo-de-libre-comercio-Mercosur-UE-en-diciembre 
la UE (residuos de industrias alimenticias y alimentos para animales; derivados minerales; oleaginosas; café, té y especias; máquinas, reactores nucleares, calderas) solo representan el $40 \%$ del comercio y muestran una mayor diversificación respecto a otros socios, lo que constituye un gran potencial para el Mercosur.

Pese a la contracción en el valor de las exportaciones, por primera vez desde el año 2012, el Mercosur muestra en el año 2016 un saldo comercial favorable de alrededor de 382 millones de dólares, aunque el monto es marginal respecto a los valores registrados hasta 2011, que se situaban por encima de los tres mil millones de dólares. La recuperación de un saldo favorable podría ser potenciada, si se lograse un acuerdo equilibrado, simétrico y progresivo.

Las razones de la UE para la conclusión de un acuerdo han sido presentadas en el análisis de sensibilidad difundido por la Comisión en el 2016 por el cual informa las siguientes "ganancias potenciales": acceso más sencillo y privilegiado a un mercado atractivo y amplio, ventajas comparativas significativas en el mercado del Mercosur, ahorros arancelarios por más de 4.000 millones de euros; acceso a materias primas esenciales a precios más competitivos, acceso preferencial a un mercado de servicios promisorio, igualdad con compañías locales en compras gubernamentales.

En paralelo a este proceso, desde 2016 Mercosur y la Asociación Europea de Libre Comercio (EFTA, por sus siglas en inglés) se encuentran consensuando los términos para una negociación de un tratado de libre comercio. EFTA es una zona de libre comercio conformada por Islandia, Liechtenstein, Noruega y Suiza, que actualmente cuenta con 27 acuerdos de libre comercio que comprenden a 38 países y se encuentra en negociaciones con al menos diez contrapartes. Este proceso se produjo, acelerado por cuestiones políticas, meses después del Brexit y luego del primer intercambio de ofertas de acceso a mercado con la UE.

\subsection{Negociaciones Mercosur - Alianza del Pacífico}

La Alianza del Pacífico (AP) fue establecida en el año 2012 a través de un Acuerdo Marco suscrito por los presidentes de Colombia, México, Chile y Perú, por el que se comprometen a construir un área de integración profunda para avanzar progresivamente a la libre circulación de factores productivos, así como convertirse en una plataforma de proyección al mundo con especial énfasis en el Asia Pacífico. Dos años más tarde se firma el Protocolo Adicional que establece una zona de libre comercio. 
Se trata de un área que reúne el 39\% del PBI de América Latina y el Caribe, suma aproximadamente el 50\% del comercio exterior y representa el $44 \%$ de los flujos de IED de la región. La Alianza se presenta también como una plataforma de acceso a mercados, con países que en promedio han suscrito alrededor de 20 acuerdos comerciales que le reportan a cada uno, y en promedio, accesos preferenciales a 57 países.

En abril de 2017 se reunieron en Buenos Aires los ministros de Relaciones Exteriores y los ministros Responsables de Comercio y Producción, acordaron una hoja de ruta común en pos de un acercamiento entre los dos bloques y la identificación de posibles cadenas regionales de valor.

Se trata de una decisión de impacto para el Mercosur, que no solo podría estrechar sus vínculos comerciales con estos países (con muchos de los cuales ya cuenta con acuerdos comerciales) sino que fundamentalmente podría complementar la tradicional asociación con el Atlántico, con una pragmática vinculación con Asia y Oceanía.

\subsection{Vinculación con China}

En el año 2015 China desplaza a la Unión Europea como el principal destino de las exportaciones del Mercosur, aunque el valor total de las exportaciones a dicho país, en concordancia con la tendencia global, desciende. La diferencia entre las exportaciones a China y a la UE fueron en 2015 de alrededor de 1700 millones de dólares, mientras que para el 2016 la diferencia se reduce a aproximadamente 610 millones de dólares, siempre a favor del gigante asiático.

Las exportaciones del Mercosur a China se encuentran en la actualidad fuertemente concentradas en unos pocos rubros, eminentemente primarios. El 86\% de las exportaciones a China en el año 2017 se concentraron en cinco rubros: oleaginosas (principalmente soja), productos minerales, bovinos, celulosa y papel, productos muy sensibles al descenso del precio de las materias primas. Por primera vez en 2016, el saldo comercial fue favorable al bloque sudamericano. 


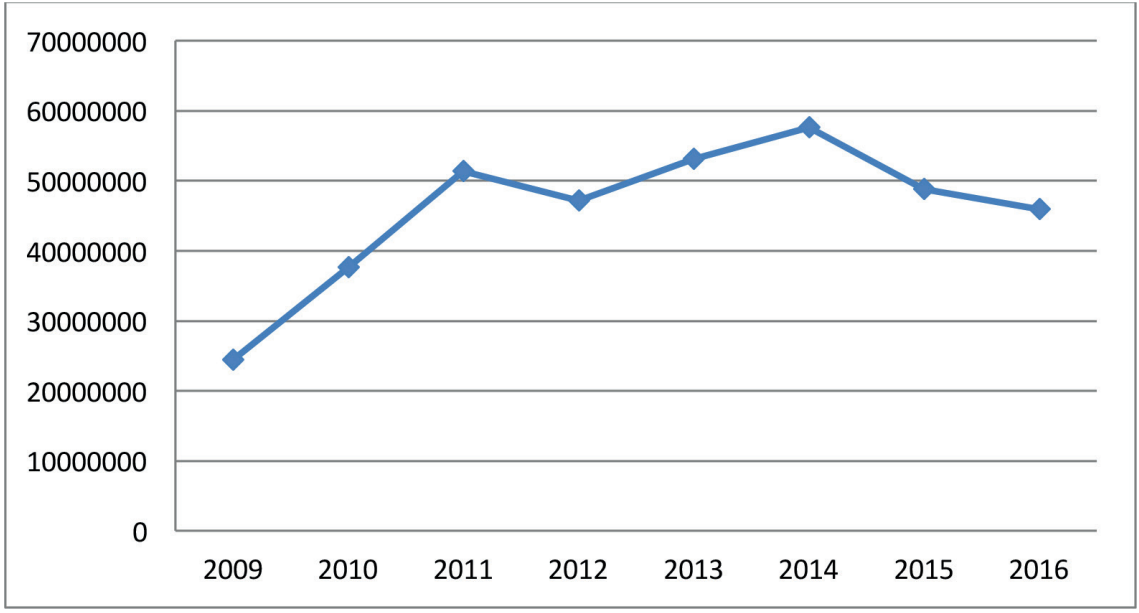

Figura 5. Exportaciones del Mercosur a la República Popular China (valor en miles de dólares). Elaboración propia sobre la base de COMTRADE (2017). Precios en dólares.

\section{El Mercosur en el nuevo sistema comercial internacional: Conclusiones exploratorias}

En los últimos quince años, el sistema internacional -y en especial los intercambios comerciales- han mutado de tal forma que la propia Comisión Europea estima que hacia 2030, el 90\% del comercio mundial se generará fuera de Europa, en especial entre países emergentes.

Tanto el ATCI como el ATP han intentado responder a este nuevo escenario mundial buscando profundizar las regulaciones vigentes en el ámbito multilateral, asegurar una inserción adecuada en las cadenas globales de valor y recuperar espacios competitivos frente a China que, junto a Rusia, son las dos grandes economías ausentes de estos esquemas. Por su parte China ha promovido un equilibrio ante estos esquemas desde la construcción del RCEP y con fuerte pragmatismo ha logrado desplazar a Estados Unidos como primer mercado para las exportaciones del Mercosur.

Como refieren Rosales, Herreros, Frohmann y García-Millán:

Las negociaciones megarregionales en curso probablemente tendrán un fuerte impacto en la distribución geográfica y la gobernanza de los flujos mundiales de comercio e inversión en los próximos años. La magnitud de 
estas iniciativas, tanto en términos del peso económico de sus participantes como de su ambiciosa agenda temática, podría en la práctica implicar que hacia el año 2020 se haya producido una verdadera redefinición de las reglas del comercio internacional. Sin embargo, a diferencia de la última gran negociación de este tipo a nivel mundial (la Ronda Uruguay del GATT, concluida en 1994), esta vez las nuevas reglas se habrían negociado enteramente fuera del ámbito multilateral y entre un número limitado de países, básicamente aquellos más vinculados a la dinámica de las redes de producción. (Rosales, Herreros, Frohmann y García-Millán, 2013, p. 47)

Estos mismos autores detectan que las consecuencias de la conclusión de estas negociaciones se medirán en términos de acceso a las cadenas globales de valor y de creación de nuevas regulaciones que incidirán también en los flujos comerciales regionales que incrementarán los niveles a los que prevalecen en las economías más desarrolladas, lo que tendría impactos sustanciales.

Sin embargo, estas negociaciones se han estancado en un escenario de sustanciales cambios geopolíticos, con un sistema comercial internacional que no recupera los niveles anteriores a la crisis de 2008 y con indicadores macroeconómicos en contracción. El análisis emprendido permite afirmar que este nuevo escenario internacional constituye una ventana de oportunidad para los países del Mercosur que, de mínima podrían mantener el status quo y, de máxima, negociar en mejores términos los acuerdos pendientes como el de Mercosur - UE, así como sus vinculaciones comerciales con la región asiática y la Alianza del Pacífico.

En ese contexto de incertidumbre el Mercosur se presenta como un socio atractivo: un mercado dinámico de 250 millones de personas que representan a nivel agregado al quinto PBI del mundo. Políticamente, es una región caracterizada por la ausencia de conflictos bélicos, aunque recurrentes crisis políticas despiertan alguna incertidumbre.

Ante este panorama, Da Motta Veiga y Rios describen las tendencias de la economía mundial que podrían actuar como desincentivos para que los principales jugadores internacionales concluyan negociaciones comerciales con los países del Mercosur, a saber:

(i) la persistente desaceleración en el crecimiento del comercio internacional; (ii) el posible sesgo proteccionista de la nueva administración estadounidense y la decisión del Reino Unido de salir de la Unión Europea; (iii) la negociación de mega-acuerdos regionales, que tienden a aumentar las exigencias, en términos de agenda temática, para que el Mercosur negocie su asociación a estos acuerdos. (Da Motta Veiga y Rios, 2017, p. 45) 
Se detecta que aunque no se ha registrado una reversión en deslocalización, el ritmo de crecimiento de la fragmentación de la producción se redujo tanto en China como en EEUU, lo que atentaría en contra de la búsqueda de nueva cadenas de valor. Analizando la propia canasta del Mercosur, sus exportaciones han estado tradicionalmente ancladas en productos agroindustriales y, a su vez, se trata de países que registran elevados niveles de protección para la importación de productos industriales, lo cual constituyen barreras por tener en cuenta en el nuevo escenario internacional. En lo que respecta a la inserción en las cadenas globales de valor y los intercambios, Fonseca y otros, afirman:

Todo esto podría afectar el posicionamiento y ascenso en la cadena de valor, especialmente la agrícola, para estos países ya sea desplazándose de dicha(s) cadena (s) y/o dejándolos en los estratos más bajos especialmente dada la mayor protección que existe en la Unión Europea y los Estados Unidos para los bienes industriales y procesados. En efecto, la posibilidad de perpetuarse como una región proveedora mayoritariamente de materias primas es uno de los mayores riesgos para los países de América Latina y el Caribe ante un posible acuerdo. (Fonseca, 2015, p. 34)

Sin embargo, en un escenario de negociaciones megarregionales estancadas, el Mercosur cuenta con una serie de oportunidades que podrían significar un avance sustancial para el futuro de un bloque que ha registrado resultados dispares en un cuarto de siglo de existencia.

Primero. El Mercosur no formó parte de las negociaciones megarregionales descriptas, por lo cual las mejoras de acceso a mercado obtenidas, a partir de la reducción de barreras arancelarias y no arancelarias para sus miembros, pudiesen haber creado externalidades que se hubiesen expresado en la pérdida de mercados y de relevancia de sus sectores productivos como destino de inversiones.

Tanto las importaciones como las exportaciones de servicios vienen cayendo en el Mercosur con mayor incidencia que en el resto de la región (-6\% vs $-1,3 \%$ en el primer trimestre de 2016). También son superiores a la media de América Latina y el Caribe las tasas de caída de exportaciones e importaciones de bienes para el 2016. La contracción hubiese sido mayor con el TPP y el TTIP en vigor (Cepal, 2016).

La Cepal (2016) estima que uno de los principales efectos del ATP hubiese sido la mayor competencia que enfrentarían las exportaciones de todos los países latinoamericanos en el mercado estadounidense. Específicamente, Argentina, Brasil y Uruguay hubiesen perdido competitividad en el mercado estadounidense frente a Nueva Zelandia en productos como carne, almidones y féculas, 
lácteos y piensos. Las mayores caídas en las exportaciones las hubieran soportado Brasil y Venezuela.

En relación con el impacto del ATCI en el Mercosur, un acuerdo sobre estándares comunes que permitieran a terceros países cumplir con normas uniformes de exportación para sus mercados podría, a largo plazo, resultar beneficioso al reducir los costos de adaptación a distintas regulaciones burocráticas. Sin embargo, en el corto plazo, obligaría a los países a modificar abruptamente sus procedimientos de exportación, lo que demandaría costos de adecuación.

En el corto plazo, también, y si estos acuerdos entrasen en vigencia, el Mercosur podría experimentar una mayor competencia en ambos mercados. Los rubros más desplazados de la cartera de exportación podrían ser vinos, bovinos y alimentos procesados.

Segundo. Al compás de los cambios políticos ocurridos desde el 2015, se han revitalizado las negociaciones pendientes entre el Mercosur y la Unión Europea, comprometiéndose las partes a suscribir un acuerdo antes del cambio de la Comisión en el año 2019. El impacto de la desgravación arancelaria, pero también de los acuerdos en materia de servicios, compras gubernamentales, propiedad intelectual, etc., sería primordial como se ha argumentado en las secciones anteriores. La concentración exportadora es mayor ante China que ante la UE, por lo cual la nueva coyuntura favorable a Europa se torna en una oportunidad de potenciar las ventas a un mercado diversificado

Tercero. Acompañando el acuerdo con la UE, que es la negociación más importante en la que está inmerso, el Mercosur ha avanzado en vinculaciones con la Alianza del Pacífico, que podrían profundizarse creando una nueva dinámica Atlántico-Pacífico. Asimismo, la política del bloque hacia la nueva administración norteamericana ha sido caracterizada por un fuerte pragmatismo. Un país del Mercosur, la República Argentina, detenta en el 2017/18 la Presidencia de la OMC y del G20.

En suma, el tiempo ganado por los cambios de contexto obliga al Mercosur a emprender un proceso de reflexión profunda intra bloque sobre qué tipo de regionalismo se implementará en esta nueva fase. Los indicadores relevados señalan que existe una coyuntura favorable a que el Mercosur se inserte competitivamente en las cadenas globales de valor. La forma en la que dicho bloque sudamericano encarará sus vinculaciones comerciales y la composición de su estructura productiva tendrán profundos impacto en la posibilidad de esa inserción. 


\section{Referencias}

Acuerdo Marco de la Alianza del Pacífico. (2012). Recuperado de https://alianzapacifico.net/documentos/

Balassa, Bela. (1982). Teoría de integración económica. México: Unión Tipográfica Editorial Hispanoamericana (UTEHA).

Baldwin, Richard. (2004). Stepping stones or building blocks? Regional and multilateral integration. G-20 Workshop on "Regional economic integration in a global framework" (Beijing: 22-23 September 2004).

Banco Mundial. (2017). Sitio oficial del Banco Mundial. Recuperado de http://data. worldbank.org/

Beattie, Bryain. (2 de mayo, 2017). Arbitration on trial: the US and UKs fear of the supranational. Financial Times. Recuperado de https:/www.ft.com/content/ e607c6b2-28f5-11e7-bc4b-5528796fe35c

BID. (2017). Tratados Comerciales en América Latina. Módulo I. ¿Qué son los acuerdos comerciales y cuál es su importancia? Nuevas tendencias en los tratados comerciales en América Latina. Autor.

Bouzas, Roberto y Zelicovich, Julieta. (Septiembre-diciembre, 2014). La Organización Mundial del Comercio, los acuerdos megarregionales y los usos estratégicos del regionalismo. Estudios de Economía Aplicada, 32(3), 963-994.

Carrión Fonseca, Gloria. (2014). El Acuerdo de Asociación Comercial y de Inversiones entre la Unión Europea y los Estados Unidos: Retos y oportunidades para las relaciones birregionales entre la UE y América Latina y el Caribe. Hamburgo. Fundación Eu-Lac. Recuperado de https:/eulacfoundation.org/ es/system/files/EU_LAC_TTIP_SPAN_WEB_150124_RZ-2\%20copy.pdf

Cepal. (2015). América Latina y el Caribe y China: Hacia una nueva era de cooperación económica. Santiago: Naciones Unidas.

Cepal. (2016). Panorama para la inserción internacional de América Latina y el Caribe. La región frente a las tensiones de la globalización. Santiago: Naciones Unidas.

Comisión Europea. (2015). Comercio para todos: Hacia una política de comercio e inversión más responsable. Bruselas: Autor. Recuperado de http://trade. ec.europa.eu/doclib/docs/2016/january/tradoc_154134.pdf

Comisión Europea. (2015). Comprehensive Free Trade Agreement with Mercosur. Potential Gains for the European Union. Bruselas: Autor. Recuperado de http://trade.ec.europa.eu/doclib/docs/2016/may/tradoc_154559.pdf (8 de agosto de 2017) 
Comtrade. (2017). Trade statistics for international business development. Recuperado de http://www.trademap.org/Index.aspx

Ecorys. (2017). SIA in support of the negotiations on a Transatlantic Trade and Investment Partnership (TTIP). Brussels: European Commmission.

Estevadeordal, Antoni. (9 de marzo, 2016). El significado del TPP para América Latina y el Caribe. Brookings. Recuperado de https:/www.brookings.edu/es/ opinions/el-significado-del-tpp-para-america-latina-y-el-caribe-2/

González, Julio. (25 de abril, 2017). Trump y el TTIP. Global Politics and Law. Recuperado de https:/www.globalpoliticsandlaw.com/2017/04/25/resurreccion-del-ttip/

Horn, Henrik, Mavroidis, Petros, y Sapir, André. (2010). Beyond the WTO? An anatomy of EU and US preferential trade agreements. The World Economy, 33, 1565-1588. Bruselas: Bruegel Blueprint Series.

Da Motta Veiga, Pedro y Ríos, Sandra. (2017). El nuevo escenario global. Los futuros del Mercosur: Nuevos rumbos de la integración regional. Nota técnica N. ${ }^{\circ}$ IDB-TN-1263.

Mercosur. (1991). Tratado para la Constitución de un Mercado Común entre la República Argentina, la República Federativa de Brasil, la República del Paraguay y la República Oriental del Uruguay. Autor.

Organización Mundial del Comercio (OMC). (1995). Acuerdo general sobre el comercio de servicios. Ginebra: OMC.

Organización Mundial del Comercio (OMC). (1994). Acuerdo de Marrakech por el que se establece la Organización Mundial del Comercio. Autor.

Organización Mundial del Comercio (OMC). (1994). Acuerdo general sobre aranceles aduaneros y comercio. Ginebra: OMC.

Rensmann, Thilo. (2017). Mega-Regional Trade Agreements, Springer.

Rosales, Osvaldo, Herreros, Sebastián, Frohmann, Alicia, García-Millan, Tania. (2013). Las negociaciones megarregionales: Hacia una nueva gobernanza del comercio mundial. Serie Comercio Internacional $N .^{\circ}$ 121. Cepal.

Tello, Mario. (2015). Cadenas globales de valor: La maldición de los recursos naturales revisados. Revista Integración y Comercio, n 39, BID-INTAL.

Warren, Elizabeth. (25 de febrero, 2015). The Trans-Pacific Partnership clause everyone should oppose. The Washington Post. Recuperado de https://www. washingtonpost.com/opinions/kill-the-dispute-settlement-language-in-thetrans-pacific-partnership/2015/02/25/ec7705a2-bd1e-11e4-b274-e5209a3bc9a9_story.html?utm_term $=.70 \mathrm{c} 7936 \mathrm{aa} 738$ 